\title{
FIBER OPTIC THERMOGRAPHIC DETECTION OF FLAWS IN COMPOSITES
}

\author{
Meng-Chou Wu and William P. Winfree \\ NASA, Langley Research Center, MS 231, Hampton, VA, USA 23681-2199
}

\begin{abstract}
Optical fibers with multiple Bragg gratings bonded to surfaces of structures were used for thermographic detection of subsurface defects in structures. The investigated structures included a 10-ply composite specimen with subsurface delaminations of various sizes and depths. Both during and following the application of a thermal heat flux to the surface, the individual Bragg grating sensors measured the temporal and spatial temperature variations. The obtained data were analyzed with thermal modeling to reveal particular characteristics of the interested areas. These results were found to be consistent with the simulation results.
\end{abstract}

Keywords: Fiber Bragg Grating, Temperature Sensor, Thermography, Composite, Delamination PACS: $72.80 . \mathrm{TM}, 42.81 . \mathrm{Wg}$

\section{INTRODUCTION}

Fiber optic sensors have been extensively studied and proposed for temperature and strain sensing in heath monitoring systems of aerospace structures and materials as well as many other applications [1-3]. Compared to other sensors, Fiber optic sensors have the advantages of being lightweight and flexible, and requiring simpler wiring especially for distributed sensing. For an extensive heath monitoring system distributed sensors are an important requirement. Most efforts have focused on using distributed fiber optic strain sensing systems, with limited consideration given to temperature sensing systems. For fiber optic temperature sensing, the conventional techniques using Raman scattering can detect the average temperature over a long distance within a single fiber. However the spatial resolutions for temperature readings-averaging over a length of fiber-are typically about one meter. Therefore they are not suitable for measuring the thermal responses at specific locations of the investigated materials.

Another more attractive alternative is using fiber Bragg gratings (FBGs) for distributed temperature sensing (DTS). FBGs written in an optical fiber have a physical length of only a few millimeters. This size is nearly a point sensor. However, if multiple FBGs are written at different (multiplexing) wavelengths and read with a time domain demodulation system, the total number of these FBGs in a singe fiber is limited due to the finite bandwidth of the laser in the detector system. To solve the above problems of spatial resolution and limited sensors, recently a new technique for fiber optic DTS has been developed using low reflectivity FBGs. Under this technique low reflectivity FBGs were 
written at the same nominal wavelength and read with a frequency domain demodulation system [4.5]. This technique allows a single fiber to contain hundreds of FBGs, employed as temperature and strain sensors.

An effective fiber optic DTS system has some advantages compared to a distributed strain sensing system for structure health monitoring. For a strain sensing system, in general, it is required to mechanically excite (or stress) the investigated structure or material [6]. For some cases this is impractical, if not impossible. Obviously, using a DTS system requires the investigated material to be thermally excited. However, the temperature change of the material from the required thermal excitation can be only a few degrees or no more than tens of degrees. Such temperature variation is comparable with the temperature changes excited for conventional thermography techniques.

Conventional thermographic techniques in general utilize a flash or quartz lamp as a heating source and an infrared imager to detect the thermal response of the investigated material. The heating pulse duration typically ranges from a fraction of a second to a few seconds. The IR imager contains an array of several hundred by hundred detectors. These techniques are capable of large area inspection of aerospace structures and materials for their reliability and safety. One of the particularly important areas is the inspection of graphite fiber reinforced composite materials because they are being increasingly used as primary structures due to their high stiffness and strength to weight ratio. Of particular interest is the detection of delaminations that can appreciably reduce the compressive strength of a composite. Recently Winfree et al. have successfully developed a physical model of two-layered systems for accurate reduction of the temporal thermal response of a composite with fabricated delaminations to the depths of the delaminations [7].

In this paper we propose a new technique using a single optical fiber with multiple FBGs for the thermographic detection of flaws in materials and structures. The investigated structures included a 10-ply composite specimen with fabricated delaminations of various sizes and depths, similar to the one mentioned above. The optical fiber was bonded to the surface of the investigated composite. Both during and following the application of a thermal heat flux to the surface, the individual Bragg grating sensors measured the temporal and spatial temperature variations. The data obtained from individual FBGs were analyzed with thermal modeling to reveal particular characteristics within the area of interest.

\section{THEORY}

\section{FBG as a Temperature Sensor}

In general a fiber Bragg grating can be characterized by its Bragg wavelength, which is the center wavelength of the light reflected from the grating. The Bragg wavelength is given as, $\lambda_{B}=2 n_{\text {eff }} \Lambda$, where $n_{\text {eff }}$ is the effective refractive index of the fiber core and $\Lambda$ the grating period. For a fiber Bragg grating bonded onto or embedded in a polymeric substrate, a change in the temperature causes a change in the grating period due to not only the thermal expansion of the fiber but also the strain induced by thermal expansion of the substrate. In addition, the refractive index of the fiber core changes because of the thermo-optic effect. Combining all the above effects, the shift in the Bragg wavelength due to a temperature change is given as

$$
\delta \lambda_{\mathrm{B}} / \lambda_{\mathrm{B}}=\left(1-\mathrm{p}_{\mathrm{e}}\right) \delta l / l+\delta \mathrm{n}_{\mathrm{eff}} / \mathrm{n}_{\mathrm{eff}}
$$


where $\mathrm{p}_{\mathrm{e}}$ is the photoelastic constant of the optical fiber and $\delta l / l$ is the thermally induced strain of the fiber. In general the photoelastic constant, the fractional index change, and the thermal expansion coefficients of the fiber and the substrate are temperature dependent and nonlinear, especially at low temperatures. However, for a finite temperature change, $\Delta T$, especially for the temperatures around and above the room temperature, Eq. (1) can be rewritten as a linear form as [8]

$$
\delta \lambda_{\mathrm{B}} / \lambda_{\mathrm{B}}=\left[\left(1-\mathrm{p}_{\mathrm{e}}\right) \alpha_{\mathrm{p}}+\xi\right] \Delta T,
$$

where $\xi=\left(1 / \mathrm{n}_{\mathrm{eff}}\right)\left(\partial \mathrm{n}_{\mathrm{eff}} / \partial T\right)$, is the thermo-optic coefficient of the fiber and $\alpha_{\mathrm{p}}$ is the thermal expansion coefficient of the substrate. Eq. (2) takes into account the conditions when the thermal expansion coefficient and the physical dimension of the substrate are much greater than those of the optical fiber. This simple equation allows the FBG to perform as a temperature sensor of the substrate.

\section{Determination of Delamination Depth from the Theoretical Thermal Response of two- layered system}

Two steps are required to determine the delamination depth from the thermal response of a composite. The first step is the development of a model for the thermal diffusion in the specimen. For this case, a one-dimensional model is proposed in the following section. After development of a model, the thermal data must be compared to different model responses to determine which model parameters give the best approximation of the data.

Real delaminations in composites are air gaps between two layers in the composite. It is well known that for long times, the response of the temperature over the delamination is not characteristic of a single layer response. The typical explanation for this effect is that lateral heat flow around the delamination diffuses the heat to the backside of the delamination. However, if the thickness of the air gap is such that the heat flow across the gap is much larger than the heat flow around the delamination, the gap's thermal resistance dominates the time response of the delamination. Note that if the thermal resistance of the delamination is sufficiently small, the time response at the front surface may not be significantly different than a single layer response.

A simple analytic solution does not exist for the one-dimensional heat flow in a multilayered material. A solution does however exist in Laplace space for two layers of thickness $l_{1}$ and $l_{2}$ coupled by an intermediate contact resistance $(R)$. Since the configuration of interest is a composite with a delamination, the first and second layers are assumed to have the same thermal conductivity $(K)$ and diffusivity $(\kappa)$. For the surface with the incident heating, the Laplace transform of the temperature response is given by [7]

$$
T_{f}(p)=\frac{f(p)\left(\sinh \left(l_{1} q\right) \sinh \left(l_{2} q\right)+\cosh \left(l_{1} q\right)\left(\cosh \left(l_{2} q\right)+K q R \sinh \left(l_{2} q\right)\right)\right)}{K q\left(\sinh \left(l_{1} q\right) \cosh \left(l_{2} q\right)+\left(\cosh \left(l_{1} q\right)+K q R \sinh \left(l_{1} q\right)\right) \sinh \left(l_{2} q\right)\right)}
$$

where $\mathrm{q}$ is $\sqrt{p / \kappa}, p$ is the coordinate in Laplace space and $f(p)=f_{0}\left(1-e^{-p t_{0}}\right) / p$, where $f_{0}$ is the energy per area from the heating source and $t_{0}$ is the heating duration.

The parameter of interest in this study is the depth of a delamination. The depth of a delamination as measured from the front surface is $l_{1}$ in Eq. (3). Since the depth always appears with diffusivity in the specimen, it is not possible to obtain a value of the depth from the thermal response without an independent measurement of diffusivity. It is 
however possible to determine the relative depth of a delamination, if there is a clear region of the composite where no delamination exists. Representing the relative depth of the delamination as $r,\left(l_{1}=r l\right.$ and $\left.l_{2}=(1-r) l\right)$, Eq. (3) can be rewritten as

$$
T_{f}(p)=\frac{a(p)\left(2 \cosh \left(\sqrt{\frac{p}{\kappa_{l}}}\right)+\sqrt{\frac{p}{\kappa_{l}}} R_{K}\left(\sinh \left(\sqrt{\frac{p}{\kappa_{l}}}\right)-\sinh \left(\sqrt{\frac{p}{\kappa_{l}}}-2 \sqrt{\frac{p}{\kappa_{l}}} r\right)\right)\right)}{\sqrt{p}\left(2 \sinh \left(\sqrt{\frac{p}{\kappa_{l}}}\right)+\sqrt{\frac{p}{\kappa_{l}}} R_{K}\left(\cosh \left(\sqrt{\frac{p}{\kappa_{l}}}\right)-\cosh \left(\sqrt{\frac{p}{\kappa_{l}}}-2 \sqrt{\frac{p}{\kappa_{l}}} r\right)\right)\right)},
$$

where $a(p)$ is $\sqrt{\kappa} f(p) / K, R_{\mathrm{K}}$ is $R K / l$, and $\kappa_{l}$ is the diffusivity of the specimen divided by the full thickness squared. $\kappa_{l}$ is estimated from a fit of the region of the specimen where there is no delamination.

With this parameterization of the equation, it is possible to find the relative depth of a delamination. Before finding the delamination depth, the characteristic $\kappa_{l}$ is determined from either a single-side or two-sided diffusivity measurement for the whole specimen. This enables both an estimate for $\kappa_{l}$ and a good image for identification of delaminations. The characteristic $\kappa_{l}$ is estimated by taking the median of the values for $\kappa_{l}$. For a longer duration heating from a quartz lamp, the thermal responses at sensor locations can be calculated by convolving these impulse responses.

\section{EXPERIMENT}

The low (smaller than a few tenths of one percent) reflectivity FBGs used in this research were written in situ, into the drawn optical fiber using the NASA Langley optical fiber draw tower. A pulsed $\mathrm{KrF}$-excimer laser of $248 \mathrm{~nm}$ and a Talbot interferometer arrangement mounted on the tower were used to write the FBGs. An aperture placed in the laser beam path was used to control the grating length and spatial profile [9]. FBG lengths for the present study were nominally $5 \mathrm{~mm}$. These single-mode fibers were drawn from commercially available germanium-doped preforms of high numerical apertures. They were coated with polyimide to thicknesses ranging from 11 to 16 micrometers, and then ink-marked to show grating locations.

These low reflectivity FBGs were interrogated using a frequency domain demodulation system shown in Fig. 1. In this system, the fiber coupler $\mathrm{C}_{1}$ and a pair of Faraday rotation mirrors (FRMs) form an in-fiber interferometer with an optical path difference of $2 n_{\text {eff }} L_{0}$, where $n_{\text {eff }}$ is the effective refractive index of the fiber core and $L_{0}$ the length of the reference cavity. The signals are driven by the tuning of the laser and detected at the photo-detector $\mathrm{D}_{1}$. They are used to trigger the sampling of signal at $\mathrm{D}_{2}$, which is the output of another in-fiber interferometer formed with the fiber coupler $\mathrm{C}_{2}$, a broadband reflector, and a particular fiber Bragg grating at a distance of $\mathrm{L}_{\mathrm{i}}$. If there is a series of low reflectivity Bragg gratings written at the same wavelength on a single fiber at different locations, the reflected signals from each grating are superimposed and detected at $\mathrm{D}_{2}$. The detected signals are fast-Fourier-transformed to obtain the spatial spectrum of all gratings, which displays the physical profiles of the gratings at different locations. The spatial spectrum of a particular grating can then be windowed and inverse-fast-Fouriertransformed to get its own wavelength spectrum. Fig. 2(a) shows the spatial spectrum of two FBGs in a fiber with multiple gratings. Two lines in Fig. 2(a) indicate the window of the inverse FFT for the second FBG. Its calculated wavelength spectrum is shown in Fig. 2(b). For the distributed thermal sensing, the center wavelength shifts of each FBG were measured and calculated using the process described above. 


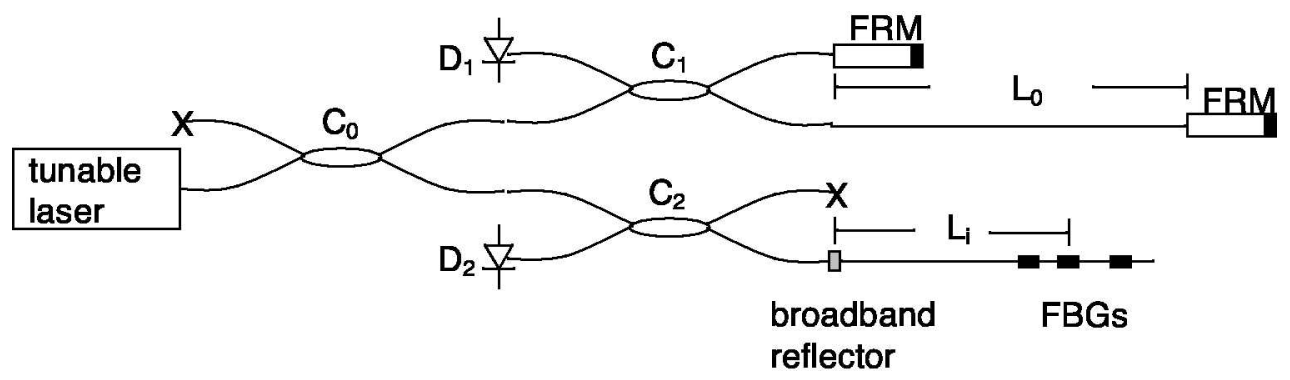

FIGURE 1. Schematic diagram of a frequency domain demodulation system. Items $\mathrm{C}_{0}, \mathrm{C}_{1}$, and $\mathrm{C}_{2}$ are fiber couplers. " $X$ " indicates that the unused port is terminated. Items $D_{1}$ and $D_{2}$ are detectors and FRMs are Faraday rotation mirrors.
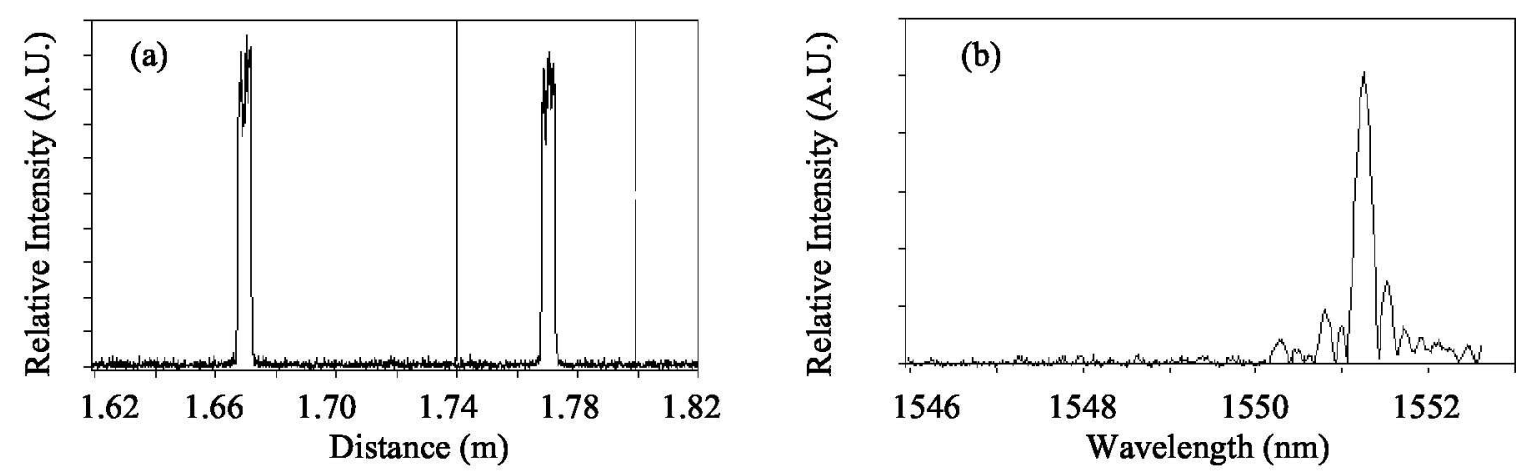

FIGURE 2. (a) The spatial spectrum of two FBGs with a physical length of about $5 \mathrm{~mm}$ and two lines indicating the window of the inverse FFT for the second FBG. (b) The calculated wavelength spectrum of the second FBG with a center wavelength of $1551.3 \mathrm{~nm}$.

In this study, an optical fiber with 32 FBGs was bonded to an investigated composite specimen with Kapton $($ tape, as shown in Fig 3 (a). This bonding technique allowed the optical fiber to be bonded and taken off the sample quickly and neatly. At the same time it also allowed the fiber to sense the thermal strain for a temperature change up to $50^{\circ} \mathrm{C}$ without imminent slipping.

The specimen used for testing the viability of this measurement technique was a composite specimen with 20 delaminations at specified depths. The 10 ply quasi-isotropic composite panel with a lay-up of $[0,45,90,-45,0 /, 45,90,-45 /, 0,90 /]$ was $31.75 \times 31.75$ centimeters and 0.19 centimeters thick (Fig. 3(a)). The delamination defect areas were squares with sizes of $3.8 \times 3.8,2.5 \times 2.5,1.9 \times 1.9$, and $1.3 \times 1.3$ square centimeters. The defects were buried at depths of $10,20,30,40$, and 50 percent of the total thickness. A schematic of the defect layout and FBG sensor numbers is shown in Fig. 3 (b). The optical fiber bonded to the specimen had FBGs with a separation (center-to-center) distance of 5.08 centimeters, which was the same as the separation distance of the squares in the same row. One FBG was placed at the center of each square. Five FBGs numbered 14-19 were outside the squares for comparison.

A quartz lamp was used to heat the front surface of the specimen. The heating durations were typically about $10-15$ seconds. The grating data acquisition was performed during and following the application of the heat flux to the surface at a rapidity rate of about $2.85 \mathrm{~Hz}$. An IR camera was also used to record the temperatures of the surface separately. The experimental setup is shown in Fig. 4. 


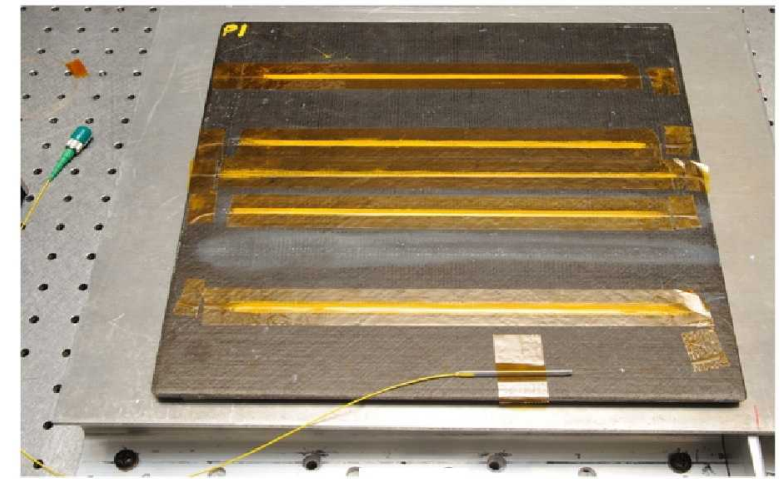

(a)

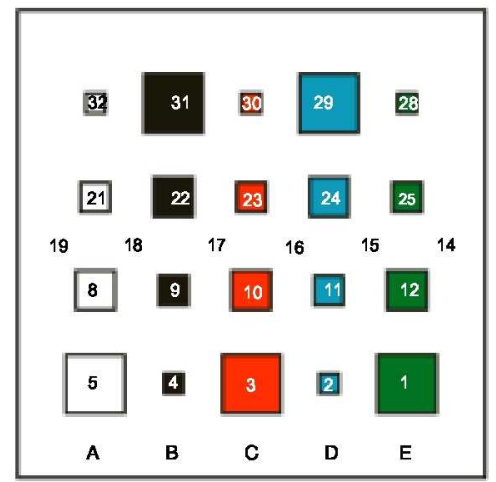

(b)

FIGURE 3. (a) A single optical fiber with multiple Bragg grating sensors bonded onto the surface of a 10ply-composite. (b) The fabricated delaminations of various sizes arranged in columns between plies 1-2 (A), 2-3 (B), 3-4 (C), 4-5 (D), 5-6 (E).

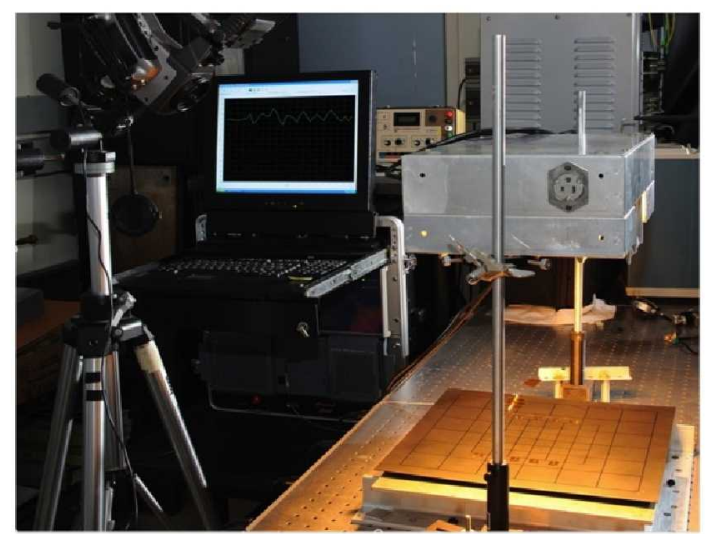

FIGURE 4. The experimental setup of the fiber optic thermal health monitoring system with a quartz lamp as the heating source and a conventional IR camera for comparison.

\section{RESULTS AND DISCUSSION}

The time evolution of the temperature of the composite during the heating and cooling is a function of its three-dimensional geometry and thermal properties. For a thin composite the initial temperature response is initially a result of heat diffusion normal to the surface and is approximately one-dimensional and given by Eq. (4). As time progresses, first the in-plane heat diffusion, then the convective heat loss at the surfaces dominates the temperature response. The expected temperature response for the composite with delaminations during and after a quartz lamp heating pulse was estimated using a finite element routine.

Common literature values of thermal properties of the composite were used. The thermal conductivity perpendicular to the fibers was assumed to be $0.97 \mathrm{~W} \mathrm{~m}^{-1} \mathrm{~K}^{-1}$ and 4.85 $\mathrm{W} \mathrm{m} \mathrm{K}^{-1}$ along the fiber. The specific heat and density were assumed to be $1270 \mathrm{~J} \mathrm{~kg}^{-1} \mathrm{~K}^{-1}$ and $1490 \mathrm{~kg} \mathrm{~m}^{-1}$ respectively. A heat transfer coefficient of $8.063 \mathrm{~W} \mathrm{~m}^{-2} \mathrm{~K}^{-1}$ for convection loss at the front and back surface was used, based on the exponential decay in temperature at late times as measured by a fiber optic sensor at the center of the panel. For delaminations, $0.025 \mathrm{~cm}$ air gaps were placed at depths that corresponded to the interface between plies at positions corresponding to the squares in the schematic of the composite shown in Fig. 3(b).

In the simulation, first a short heating pulse of $10 \mathrm{~ms}$ duration was applied to the outer surface of the composite. The temperatures at the front and back surface were 
calculated at $10 \mathrm{~ms}$ intervals for the first 3.5 seconds, then at 3.5 second intervals for a total of 193 seconds. The response for quartz lamp heating was calculated at sensor locations by convolving the response from this "impulse" response, with a pulse shape that is estimated from the fiber optic sensor in the center of the panel to be a 2 second linear ramp up to a constant flux, with 13.5 second hold and a subsequent 2 second linear ramp down. Using this pulse shape, a model response at a sensor location without delamination was calculated as shown in Fig. 5(a).

As described in Experiment Section, the thermal responses of each FBG in the fiber were calculated from the measured center wavelength shifts of the FBG in a temporal sequence. Fig. 5(a) also shows the temporal thermal response of the FBG Sensor 16, located in an area without delamination. The relatively good agreement can be seen between the fiber optic sensor and the model response.

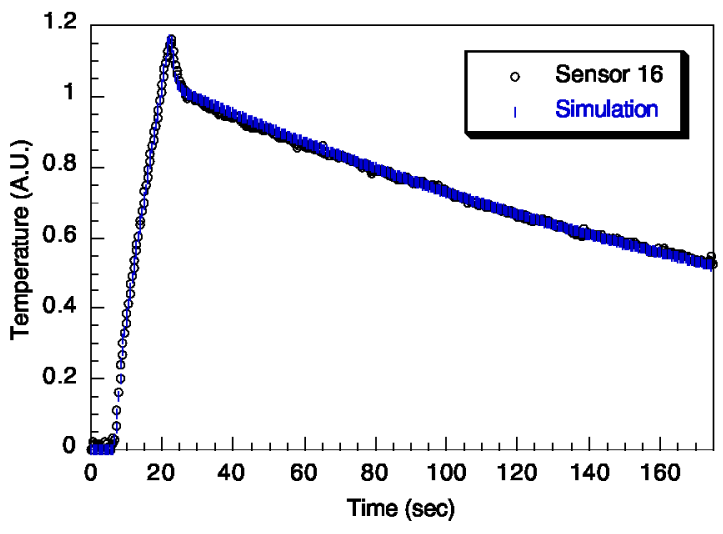

(a)

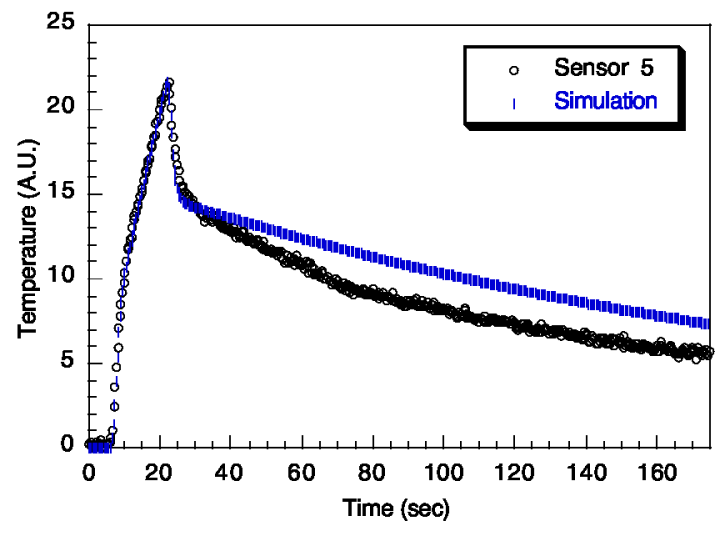

(b)

FIGURE 5. Comparison of finite element simulation to fiber optic measured temperature response at center of composite in region with (a) no delamination and (b) a delamination.

Similarly, shown in Fig. 5(b) are the simulation and the measured fiber optic temperature response for an area with a delamination depth of 10 percent of the total thickness. Both cases show good agreement between the simulation and the experimental data during and immediately following the heat pulse. At later times the temperatures measured by the fiber optic sensor significantly deviates from the simulation results. This deviation is most likely the result of uneven heating, which is not incorporated into the simulation.

By neglecting the thermal response of the later cooling stage and comparing the measured fiber optic data with the model, the depths of delaminations can be estimated. Fig. 6 shows the estimated depths of the delaminations from the fiber optic measurements for all of the $3.8 \mathrm{~cm} \times 3.8 \mathrm{~cm}$ squares (no. 1, 3,5,29, and 31, as shown in Fig. 3 (b)). The solid line represents the model values of the depths. The first one (10\% of the thickness) has the highest degree of agreement (0.91, the ratio of estimated depth/ specification depth). The rest have lesser degrees of agreement, ranging from 0.55 to 0.72 . These results are consistent with those from the conventional thermography technique applied to the same sample [7], but with greater errors, especially for the deeper delaminations.

From the current set of measurements, the depth of the smaller sizes of delaminations is more difficult to determine. Based on the simulations, the physical length of FBGs (typically $5 \mathrm{~mm}$ ) does not seem to be a limiting factor for these measurements. The depth measurement for the smaller delaminations should improve if the heat pulse duration is reduced and the frequency of the temperature measurement is increased. These 
changes will reduce the effects of uneven heating and result in a thermal response that more closely approximates the one dimensional model.

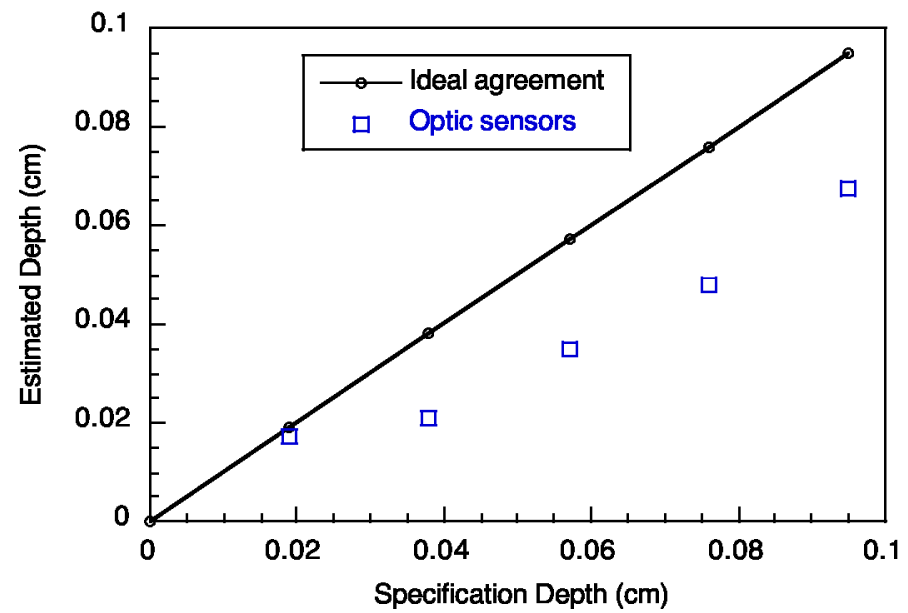

FIGURE 6. Comparison of the estimated depths of the delaminations measured by the optic sensors to the model values for all of the $3.8 \mathrm{~cm} \times 3.8 \mathrm{~cm}$ squares.

\section{CONCLUSION}

We have developed a new technique of using distibuted FBG sensors for thermographic detection of flaws in materials and structures. Individual fibers with multiple FBGs employed as temperature sensors can be bonded to the surfaces of structures or embedded in the structures. By applying a thermal heat flux to the surface of the investigated structures, the individual Bragg grating sensors successfully measured the temporal and spatial temperature variations on the surface. These thermal responses were consistent with the results from conventional thermographic techniques. We have also applied this technique on a 10-ply composite with fabricated delaminations of various sizes and depths. With different degrees of success, it has detected the square delaminations with widths of $3.8 \mathrm{~cm}$ at depths from $10 \%$ to $50 \%$ of the total thickness. Future efforts will focus on developing the technique with a faster detection system and assessing its potential for performing thermal health monitoring of aerospace structures and materials.

\section{REFERENCES}

1. C. R. Giles, J. Lightwave Technol. 15, 1391-1404 (1997).

2. K. O. Hill, Y. Fujii, D. C. Johnson, and B. S. Kawasaki, Appl. Phys. Lett. 32, 647-649 (1978).

3. A. D. Kersey, M. A Davis, H. J. Patrick, M. LeBlanc, K. P. Koo, C. G. Askins, M.A. Putnam, E. J. Friebele, J. Lightwave Technol. 15, 1442-1463 (1997).

4. M. Froggatt and J. Moore, Appl. Opt. 37, 1741-1746 (1998).

5. M.-C. Wu, R. Pater, and S. L. DeHaven, "Effects of coating and diametric load on fiber Bragg gratings as cryogenic temperature sensors," Proc. SPIE, 6933, 693303 (2008).

6. S. W. James, M. L. Dockney, and R. P. Tatam, Electron. Lett. 32, 1133-1134 (1996).

7. W. P. Winfree and J. Zalameda, "Thermographic determination of delamination depth in composites," in Thermosense XXV, Proc. SPIE 5073, 363-373 (2003).

8. S. Gupta, T. Mizunami, T. Yamao, and T. Shimomura, Appl. Opt. 25, 5202-5205 (1996).

9. M.-C. Wu and R. S. Rogowski, Appl. Opt. 42, 5017-5023 (2003). 